\title{
Panorama do licenciamento ambiental de campos de petróleo do RN
}

\author{
Eliane Patrícia de S. F. Limeira', Mariana Lopes Medeiros², Carlos Enrique de Medeiros Jerônimo³ \\ 1,2,3 Universidade Potiguar, Natal, RN, Brasil
}

\section{Resumo}

O Estado do Rio Grande do Norte é o terceiro produtor brasileiro de petróleo e gás natural sendo esta a sua principal atividade extrativista mineral. Segundo dados da PETROBRAS (2007), há reservas da ordem de 410 milhões de barris de petróleo e 133 milhões de gás natural na Bacia Potiguar. Desse modo, as atividades petrolíferas assumem vital importância para a economia do Rio Grande do Norte, sendo o recebimento de royalties, umas das principais fontes de renda do Estado e de seus municípios. O Licenciamento Ambiental é o procedimento administrativo por meio do qual se avalia a localização e se autoriza a implantação e a operação de empreendimentos considerados efetiva ou potencialmente causadores de poluição ou degradação ambiental, sendo o IDEMA, conforme Decreto nº. 14.338, de 25/02/1999, que aprovou o Regulamento deste órgão, como competente para formular, coordenar, executar e supervisionar a política estadual de preservação, conservação, aproveitamento, uso racional e recuperação dos recursos ambientais (Art. $2^{\circ}$, III). Mais especificamente, o Art. 14 diz que cabe à Coordenadoria do Meio Ambiente (CMA) do IDEMA analisar projetos e demais documentos referentes à concessão ou renovação de licença e à implantação de equipamentos e sistemas de controle de poluição. O presente trabalho tem como objetivo realizar um levantamento quantitativo referente ao Licenciamento Ambiental, para a atividade petrolífera no Estado do Rio Grande do Norte. O estudo foi realizado no Instituto de Desenvolvimento Sustentável e Meio Ambiente (IDEMA), autarquia vinculada à Secretaria de Estado do Planejamento e das Finanças (SEPLAN). O trabalho consistiu no levantamento de informações coletadas nos processos existentes no órgão ambiental do estado, através do sistema eletrônico de licenciamento ambiental do IDEMA, denominado "CERBERUS".

Palavras-chave: petróleo, cerberus, licenciamento ambiental.

\begin{abstract}
The state of Rio Grande do Norte is the third producer of oil and natural gas that is its main mineral extraction activity. According to data from PETROBRAS (2007), there are reserves of about 410 million barrels of oil and 133 million natural gas in Potiguar. Thus, oil activities are vital to the economy of Rio Grande do Norte, and receipt of royalties, one of the main sources of income of the state and its municipalities. Environmental Licensing is the administrative procedure by which to evaluate the location and permitted the establishment and operation of enterprises considered effectively or potentially causing pollution or environmental degradation, and the IDEMA as Decree. 14.338, of 25/02/1999, which approved the Regulation of this organ, responsible for formulating, coordinating, implementing and overseeing the state policy of preservation, conservation, utilization, rational use and protection of environmental resources (Art. 2, III ). More specifically, Article 14 says that it is for the Coordination of Environment (CMA) of IDEMA analyzes projects and other documents relating to the grant or renewal of license and deployment of equipment and pollution control systems. The present study aims to conduct a quantitative survey in Environmental License for the oil activity in the State of Rio Grande do Norte. The study was conducted at the Institute of Sustainable Development and Environment (IDEMA), linked to the Department of Planning and Finance (SEPLAN) authority. The work consisted of the survey collected information on existing processes in the state EPA, through the electronic system of environmental licensing IDEMA called "CERBERUS".
\end{abstract}

Keywords: Petroleum, cerberus, environmental licensing. 


\section{INTRODUÇÃO}

O Licenciamento Ambiental constitui importante instrumento de gestão do ambiente, pois é a partir dele que a Administração Pública busca exercer o controle necessário sobre as atividades humanas que interferem nas condições ambientais, de forma a compatibilizar o desenvolvimento econômico com a preservação do equilíbrio ecológico.

A preocupação com a proteção do meio ambiente hoje, é cada vez maior. A partir do momento em que o ser humano começou a sentir os efeitos de um ambiente degradado e com sérias alterações, tomou consciência de que isso implicava em diminuição representativa de sua qualidade de vida. Licenciando-se ambientalmente todos os empreendimentos efetivo ou potencialmente causador de poluição, estar-se-á promovendo a manutenção dos recursos naturais: ar, água, solo, vegetação e animais; para que as gerações futuras possam usufruir destes recursos tal como a geração anterior, assegurando a conservação do meio ambiente, e conseqüentemente, a qualidade de vida da população.

Por outro lado, tendo a Política Nacional de Meio Ambiente consagrado, dentre outros, os princípios da "racionalização do uso do solo, do subsolo, da água e do ar", do "planejamento e fiscalização do uso dos recursos ambientais" e do "controle e zoneamento das atividades "potenciais ou efetivamente poluidoras", para assegurar a sua execução, estabeleceu como um de seus principais instrumentos o licenciamento ambiental.

Sendo assim, o objetivo do licenciamento é, acima de tudo, a preservação e restauração dos recursos ambientais com vistas à sua utilização racional e disponibilidade permanente, concorrendo para a manutenção do equilíbrio ecológico propício à vida.

Entretanto, o licenciamento ambiental é imprescindível para o correto gerenciamento dos recursos naturais no Brasil, e os órgãos de fiscalização procuram assegurar que as ações que impactam o meio ambiente sejam conduzidas nos termos da legislação vigente. Embora a conscientização dos interessados e envolvidos com o assunto sobre a importância da licença tenha crescido de forma significativa nos últimos anos, verifica-se que muitas irregularidades são cometidas por falta de informação por parte dos responsáveis. Então esse poderoso instrumento proporciona ganhos de qualidade ao meio ambiente e à vida das comunidades numa melhor perspectiva de desenvolvimento.

Instituído há mais de duas décadas, o Licenciamento Ambiental, contudo, ainda enfrenta problemas que o afastam de um padrão ideal de funcionamento, isso, em grande parte, pela falta de informação adequada pela maioria dos interessados quanto aos procedimentos e trâmites requeridos para a sua concessão. Sem esses empecilhos, o licenciamento seria mais rápido e eficiente.

$\mathrm{O}$ presente trabalho tem como objetivo realizar um levantamento quantitativo referente ao Licenciamento Ambiental, para a atividade petrolífera no Estado do Rio Grande do Norte. O estudo foi realizado no Instituto de Desenvolvimento Sustentável e Meio Ambiente (IDEMA), autarquia vinculada à Secretaria de Estado do Planejamento e das Finanças (SEPLAN). O trabalho consistiu no levantamento de informações coletadas nos processos existentes no órgão ambiental do estado, através do sistema eletrônico de licenciamento ambiental do IDEMA, denominado "CERBERUS".

\section{METODOLOGIA}

O objetivo do estudo desse artigo foi realizar um levantamento quantitativo das licenças ambientais de cada atividade petrolífera emitidas entre o período de 2005 à 2013. Com isso, foi realizada uma pesquisa quantitativa de caráter descritiva.

Cleber (2013) explica que a pesquisa quantitativa considera que tudo pode ser quantificável, o que significa traduzir em números opiniões e informações para classificá-las e analisá-las.

A pesquisa descritiva, de acordo com Cervo e Bervian (2002), permite levantar as características dos fenômenos estudados e identificar as correlações entre os fatos.

Segundo Michel (2005), a pesquisa quantitativa é um método de pesquisa social que utiliza a quantificação nas modalidades de coleta de informações e no seu tratamento, mediante técnicas estatísticas, tais como percentual, média, desvio-padrão, coeficiente de correlação, análise de regressão, entre outros. Frequentemente são utilizadas, quando se necessita garantir a precisão dos resultados, evitando distorções de análise de interpretação e possibilitando uma margem de segurança quanto às inferências, ou seja, é projetada para gerar medidas precisas e confiáveis que permitam uma análise 
estatística.

Usou-se, como principal mecanismo de coleta de dados, instituições públicas, por exemplo, Instituto de Desenvolvimento Econômico e Meio Ambiente no Rio Grande do Norte (IDEMA), onde foi feito um levantamento, através do sistema eletrônico de licenciamento ambiental do IDEMA, denominado "CERBERUS".

\section{RESULTADOS E DISCUSSÃO}

Foram analisados de maneira global para este levantamento preliminar cerca de 12.500 processos requeridos ao IDEMA no período de junho de 2005 até 30 de Outubro de 2013, conforme tabelas abaixo:

Tabela 01 - Todas as Atividades petrolíferas - Retirada do Sistema CERBERUS - IDEMA

\begin{tabular}{|c|c|c|c|c|c|c|c|c|c|c|}
\hline \multicolumn{11}{|c|}{ Licenças emitidas do setor Petrolífero no período de junho de 2005 até 30 de outubro de 2013 por atividade. } \\
\hline \multirow{2}{*}{ Atividade } & \multicolumn{9}{|c|}{ Ano de emissão da licença } & \multirow{2}{*}{$\begin{array}{l}\text { Total } \\
\text { geral }\end{array}$} \\
\hline & 2005 & 2006 & 2007 & 2008 & 2009 & 2010 & 2011 & 2012 & 2013 & \\
\hline Base de armazenamento de produtos químicos & 0 & 4 & 1 & 5 & 7 & 5 & 1 & 4 & 2 & 29 \\
\hline Centro de defesa ambiental & 0 & 1 & 0 & 0 & 2 & 1 & 0 & 1 & 0 & 5 \\
\hline Complexo industrial & 0 & 1 & 0 & 0 & 8 & 3 & 4 & 2 & 6 & 24 \\
\hline Estação coletora central & 0 & 5 & 3 & 10 & 6 & 7 & 14 & 8 & 8 & 61 \\
\hline Estação coletora e compressora & 0 & 3 & 6 & 25 & 19 & 5 & 6 & 8 & 6 & 78 \\
\hline Estação coletora satélite & 0 & 30 & 23 & 64 & 48 & 25 & 37 & 29 & 48 & 304 \\
\hline Estação de fluidos & 0 & 1 & 2 & 4 & 5 & 0 & 3 & 1 & 1 & 17 \\
\hline Estação de teste & 1 & 3 & & 8 & 4 & 1 & 4 & 1 & 3 & 25 \\
\hline Estação de tratamento de óleo & 0 & 0 & 1 & 0 & 0 & 1 & 1 & 1 & 1 & 5 \\
\hline Linha de Surgência & 0 & 143 & 382 & 1045 & 819 & 428 & 477 & 568 & 447 & 4309 \\
\hline Oleoduto/gasoduto/vapor - até $10 \mathrm{~km}$ & 4 & 52 & 99 & 128 & 89 & 52 & 58 & 50 & 43 & 575 \\
\hline Poço de petróleo e /ou gás natural & 9 & 487 & 522 & 1737 & 1207 & 693 & 730 & 849 & 670 & 6904 \\
\hline Sísmica & 7 & 10 & 4 & 22 & 6 & 8 & 5 & 3 & 0 & 65 \\
\hline Sistema de injeção de água produzida & 0 & 2 & 4 & 10 & 14 & 10 & 6 & 15 & 9 & 70 \\
\hline Terminal de combustivel & 0 & 0 & 0 & 2 & 1 & 2 & 2 & 1 & 2 & 10 \\
\hline Terminal de petróleo & 1 & 1 & & 4 & 3 & 4 & 1 & 3 & 2 & 19 \\
\hline Total geral & 22 & 743 & 1047 & 3064 & 2238 & 1245 & 1349 & 1544 & 1248 & 12500 \\
\hline
\end{tabular}

Tabela 02 - Todos os Tipos de Licenças das Atividades Petrolíferas

\begin{tabular}{|c|c|c|c|c|c|c|c|c|c|c|}
\hline \multicolumn{11}{|c|}{ 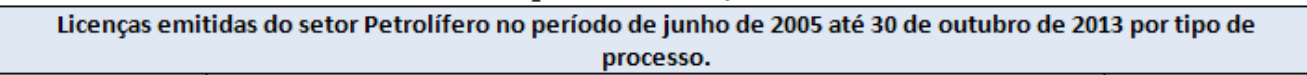 } \\
\hline \multirow{2}{*}{ Atividade } & \multicolumn{9}{|c|}{ Ano de emissão da licença } & \multirow{2}{*}{ Total geral } \\
\hline & 2005 & 2006 & 2007 & 2008 & 2009 & 2010 & 2011 & 2012 & 2013 & \\
\hline $\mathrm{AE}$ & 0 & 0 & 0 & 0 & 1 & 1 & 0 & 0 & 0 & 2 \\
\hline LA & 0 & 10 & 10 & 11 & 25 & 24 & 29 & 25 & 28 & 162 \\
\hline LI & 0 & 29 & 160 & 298 & 83 & 18 & 6 & 7 & 5 & 606 \\
\hline LIO & 0 & 0 & 0 & 41 & 56 & 54 & 67 & 57 & 60 & 335 \\
\hline LO & 13 & 133 & 457 & 661 & 486 & 315 & 148 & 117 & 116 & 2446 \\
\hline LP & 0 & 2 & 5 & 13 & 4 & 8 & 9 & 2 & 4 & 47 \\
\hline LPpe & 5 & 201 & 234 & 413 & 167 & 83 & 93 & 60 & 66 & 1322 \\
\hline LPpr & 0 & 4 & 1 & 19 & 13 & 9 & 6 & 7 & 5 & 64 \\
\hline LRO & 0 & 61 & 6 & 12 & 6 & 14 & 3 & 1 & 16 & 119 \\
\hline LS & 0 & 1 & 3 & 10 & 5 & 3 & 5 & 1 & 1 & 29 \\
\hline LSIO & 0 & 0 & 0 & 0 & 1 & 1 & 0 & 0 & 0 & 2 \\
\hline PPV & 0 & 0 & 19 & 134 & 14 & 1 & 2 & 0 & 0 & 170 \\
\hline RLA & 0 & 1 & 1 & 0 & 0 & 0 & 0 & 0 & 0 & 2 \\
\hline RLI & 1 & 3 & 3 & 0 & 0 & 0 & 0 & 0 & 0 & 7 \\
\hline RLIO & 0 & 0 & 0 & 2 & 1 & 0 & 0 & 0 & 0 & 3 \\
\hline RLO & 3 & 291 & 144 & 1444 & 1355 & 685 & 956 & 1243 & 941 & 7062 \\
\hline RLPe & 0 & 7 & 4 & 2 & & 0 & 0 & 0 & 0 & 13 \\
\hline RLS & 0 & 0 & 0 & 4 & 12 & 4 & 6 & 5 & 3 & 34 \\
\hline SVeg & 0 & 0 & 0 & 0 & 9 & 25 & 19 & 19 & 3 & 75 \\
\hline Total geral & 22 & 743 & 1047 & 3064 & 2238 & 1245 & 1349 & 1544 & 1248 & 12500 \\
\hline
\end{tabular}


Tabela 03 - Retirada da Tabela 01 - Atividades Petrolíferas que Liberam maior Quantidade de Licenças no IDEMA

\begin{tabular}{|c|c|c|c|c|c|c|c|c|c|}
\hline & 2005 & 2006 & 2007 & 2008 & 2009 & 2010 & 2011 & 2012 & 2013 \\
\hline Estação ooletora central & 0 & 5 & 3 & 10 & 6 & 7 & 14 & 8 & 8 \\
\hline Estação ooletora e compressora & 0 & 3 & 6 & 25 & 19 & 5 & 6 & 8 & 6 \\
\hline Estação ooletora satélite & 0 & 30 & 23 & 64 & 48 & 25 & 37 & 29 & 48 \\
\hline Estações coletoras & 0 & 381 & 32 & 99 & $73^{\prime}$ & 37 & $57^{\prime}$ & $45^{\prime}$ & 62 \\
\hline Atividade & 2005 & 2006 & 2007 & 2008 & 2009 & 2010 & 2011 & 2012 & 2013 \\
\hline Estações coletoras & 0 & 38 & 32 & 99 & 73 & 37 & 57 & 45 & 62 \\
\hline Atividade & 2005 & 2006 & 2007 & 2008 & 2009 & 2010 & 2011 & 2012 & 2013 \\
\hline Sistemade injeção de água produzid & 0 & 2 & 4 & 10 & 14 & 10 & 6 & 15 & 9 \\
\hline Atividade & 2005 & 2006 & 2007 & 2008 & 2009 & 2010 & 2011 & 2012 & 2013 \\
\hline Oleoduto/gasoduto/vapor- até $10 \mathrm{~km}$ & 4 & 52 & 99 & 128 & 89 & 52 & 58 & 50 & 43 \\
\hline Atividade & 2005 & 2006 & 2007 & 2008 & 2009 & 2010 & 2011 & 2012 & 2013 \\
\hline Linhade Surgência & 0 & 143 & 382 & 1045 & 819 & 428 & 477 & 568 & 447 \\
\hline Atividade & 2005 & 2006 & 2007 & 2008 & 2009 & 2010 & 2011 & 2012 & 2013 \\
\hline Pogo de petróleoe /ou gás natural & 9 & 487 & 522 & 1737 & 1207 & 698 & 730 & 849 & 670 \\
\hline
\end{tabular}

No Gráfico 1, juntamos as três Estações Coletoras: Central, Compressora e Satélite.

Observa-se que a Central obteve um número maior de licenças no ano de 2008 e 2009 e uma queda em 2013. A Compressora obteve mais licenças no ano de 2008 e 2009 e também uma queda em 2013. A Satélite ficou diferenciada das outras com um número maior nos anos de 2006, 2008, 2009 e 2013.

Gráfico 01 - Estações Coletoras: Central, Compressora e Satélite

\section{Estações coletoras}

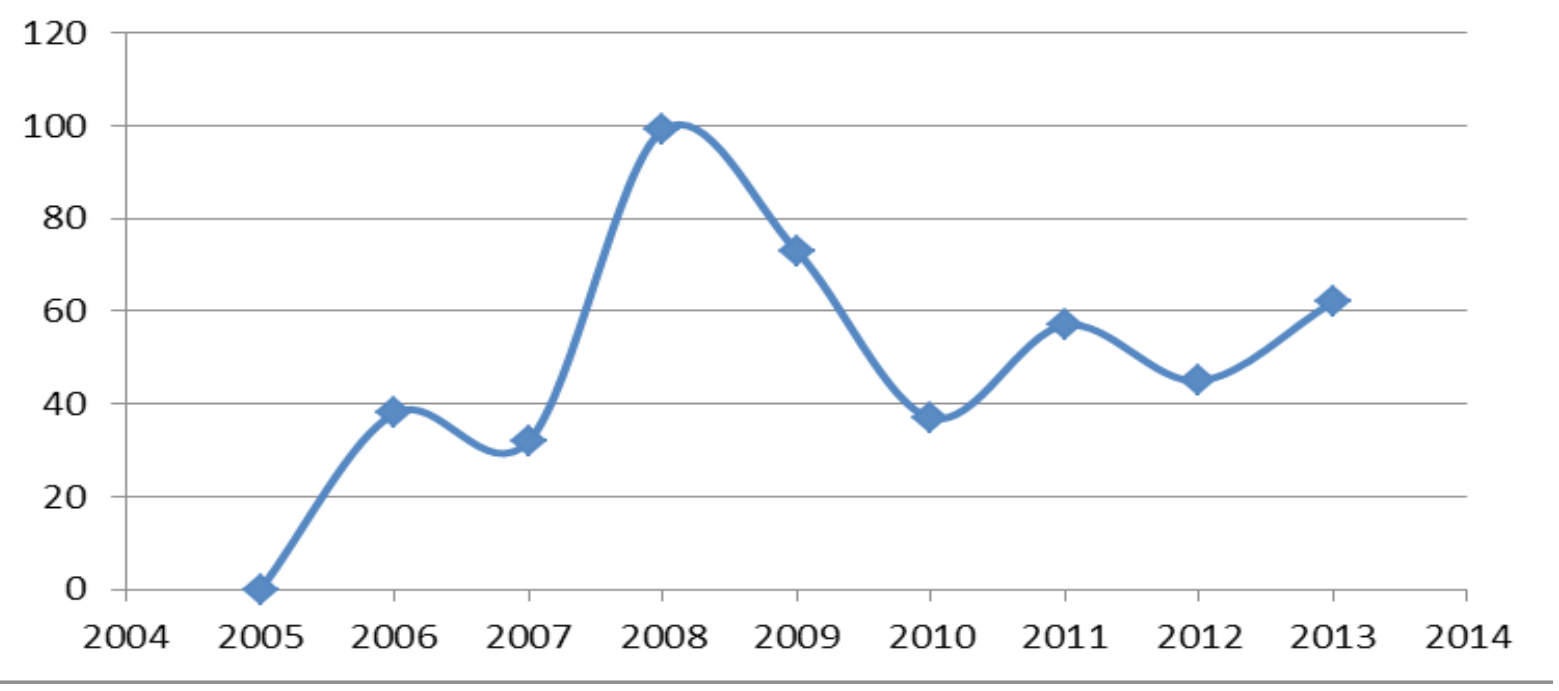


Gráfico - 02 - Sistema de Injeção de água

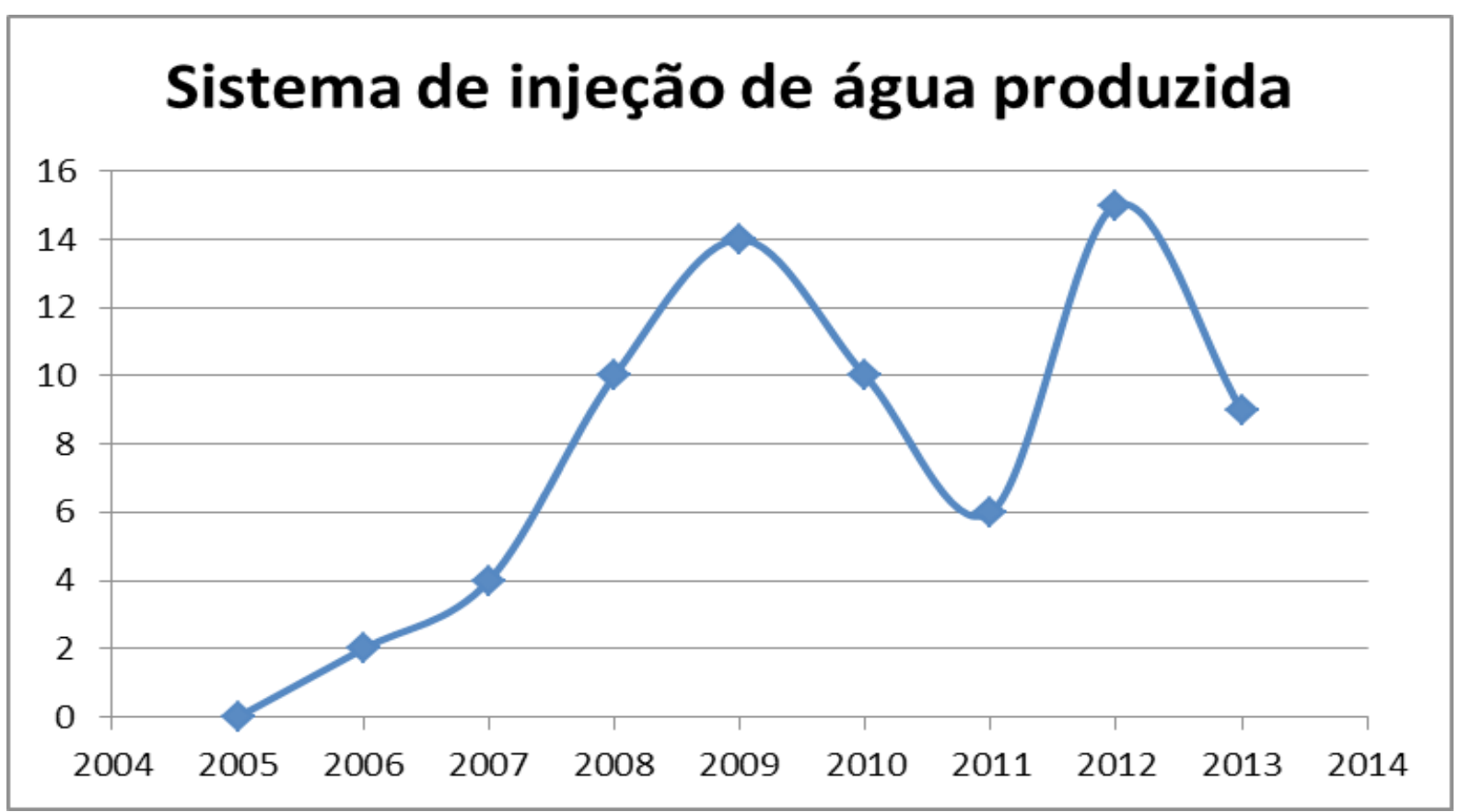

No Gráfico 02, observa-se que o maior número de Licenças obtidas foram nos anos: 2008, 2009 e 2012 e com uma queda em2013. E a maior quntidade em 2012.

No Gráfico 03, observa-se que os anos de: 2007, 2008, 2009 e 2011 tiveram um número maior de licenças e com uma queda em 2013, com um número maior em 2008

Gráfico 03 - Oleoduto e Gasoduto.

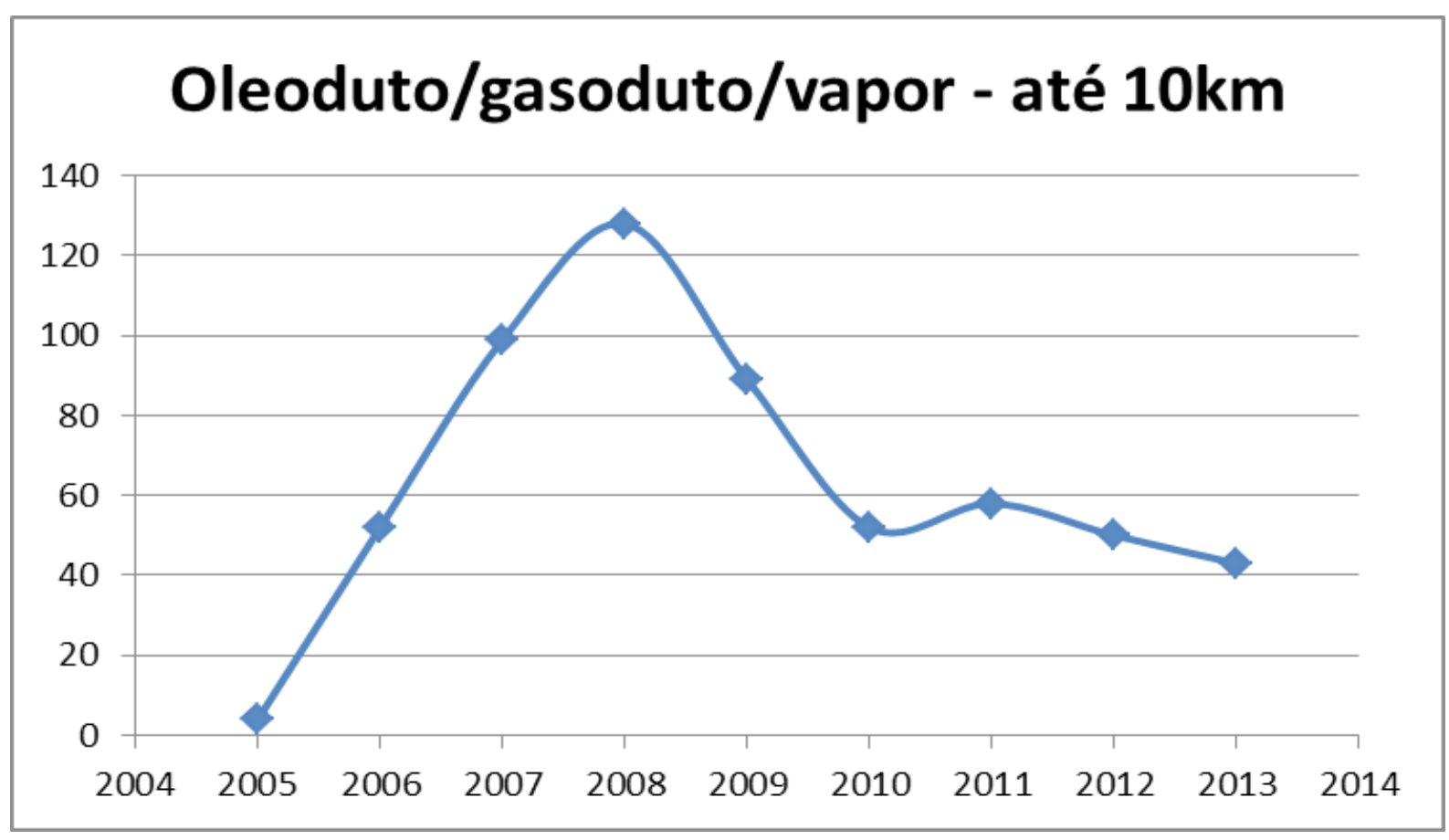




\section{Linha de Surgência}

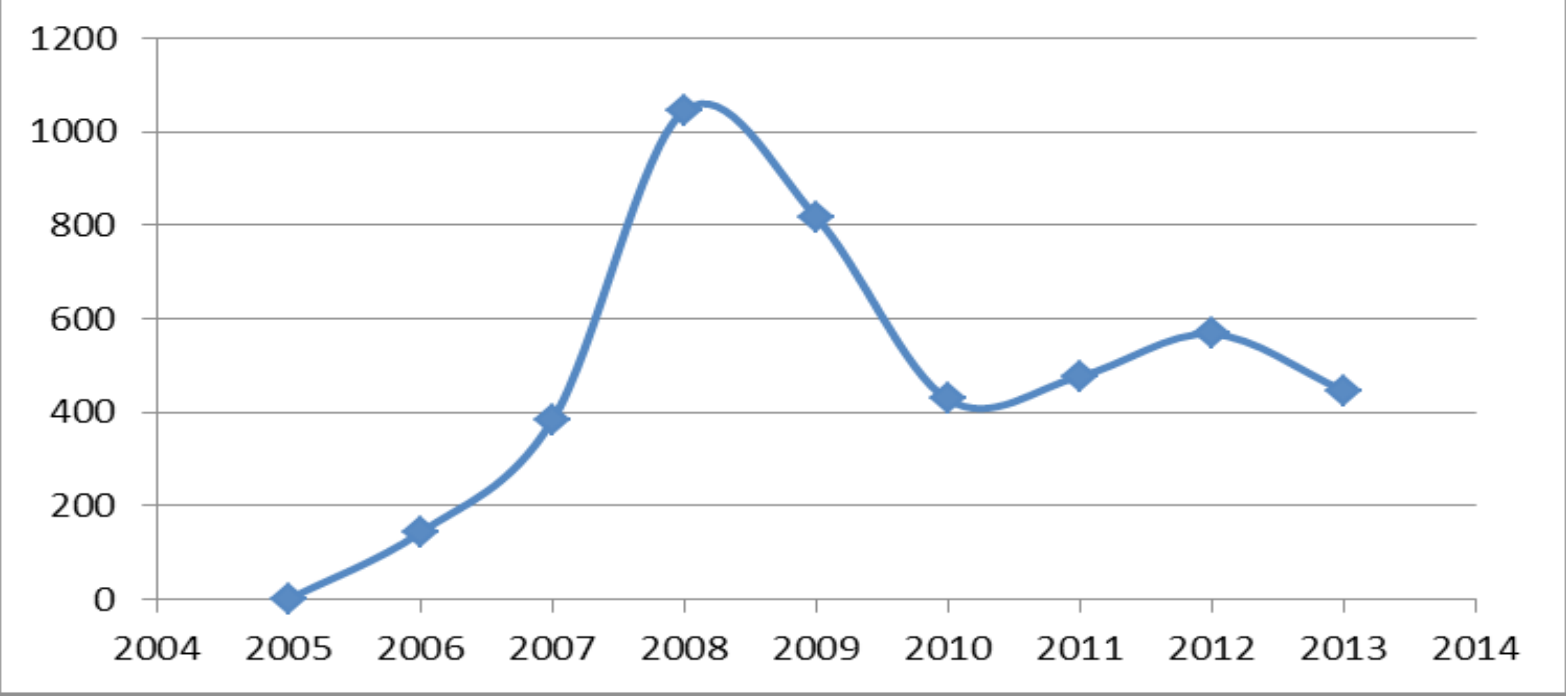

Gráfico 04 - Linha de Surgência

No Gráfico - 04, Observa-se que os anos de: 2007, 2008, 2009,2011, 2012 e 2013, obtiveram um maior número de Licenças. Mas 2008 se destacou com o número mais elevado.

No Gráfico 05, observa-se que nos anos de: 2008 e 2009 o número de Licenças emitidas foi bastante alto. Enquanto nos anos de: 2006 até 2007, ocorreu uma subida. E nos anos de: 2010 até 2011, também continuou subindo e finalmente nos anos de: 2012 até 2013, ocorreu uma queda nas Licenças.

Gráfico 05 - Poco de Petroléo ou Gás Natural

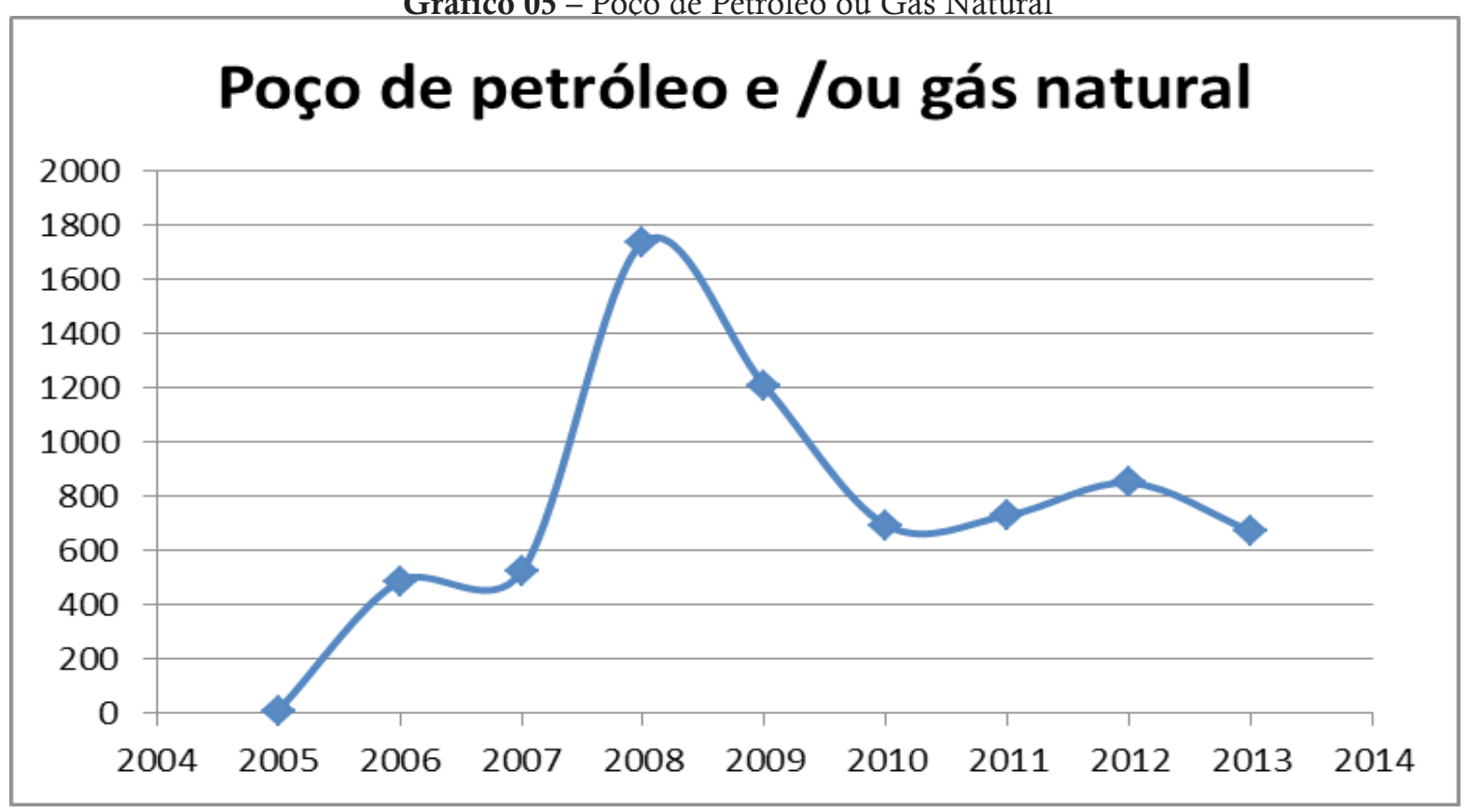


A existência da atividade petrolífera no Rio Grande do Norte faz parte da história local dos municípios produtores espalhados pelo território potiguar. As primeiras pesquisas de petróleo no estado tiveram início ainda em 1943 pelo Departamento Nacional de Produção Mineral - DNPM. Até o começo da década de 1970 prevaleceram estudos de reconhecimentos da bacia, a maioria feita pela Petrobras, utilizando geologia de superfície, métodos geológicos e perfuração de poços. (JESIEL, 2007)

A partir da década de 1970 a indústria do petróleo se instalou definitivamente no cenário potiguar, com a descoberta em 1973 do campo marítimo de Ubarana, situado na plataforma continental, tendo iniciado a sua produção a partir de 1976. Ainda em 1979, inicia-se a produção terrestre com a perfuração do primeiro poço no município de Mossoró. E sucessivamente outras descobertas terrestres nos campos de Fazenda Belém, Alto do Rodrigues, Estreito, Macau, Guamaré e Canto do Amaro - o de maior produção entre os outros. Os campos marítimos sucessores a Ubarana, no litoral Potiguar, foram Agulha, Aratum, Pescada e Arabaiana. (JESIEL, 2007)

Com o histórico de ser o pioneiro e maior produtor de petróleo em campos terrestres, o Rio Grande do Norte em 2009 rompeu a fronteira de exploração e produção e recebeu investimentos da estatal Petrobras para iniciar a construção de uma unidade de refino, criando a Refinaria Potiguar Clara Camarão. Objetivando situar a economia do Rio Grande do Norte, nesta pesquisa teórica foi realizada uma breve introdução sobre o histórico da atividade da indústria do Petróleo no estado e pesquisados os dados econômicos atuais disponíveis sobre as reservas até a distribuição de derivados. Interpretando as observações foi possível identificar que o território potiguar possui dados relevantes de reserva e produção, está desenvolvendo sua autossuficiência em relação ao abastecimento de combustíveis, possui capacidade de comercializar excedentes de produção de derivados e os recursos financeiros gerados pelo por este setor financiam o crescimento econômico da região.

O Licenciamento Ambiental das atividades petrolíferas no IDEMA é responsável por 95\% da arrecadação do orgão no estado. As licenças mais emitidas no orgão são: Licença de Operação ( LO), Renovação da Licença de Operação ( RLO), Licença Prévia de Perfuração ( LPPER) e Licença de Instalação e Operação ( LIO). Atuamente o maior número são as Renovações das licenças de operação e em seguida as Licenças de operação. E em relação as Licenças de Perfuração ouve uma queda bastante substãncial, devido aos campos que estão maduros e já produzem á cerca de 30 anos. E as Licenças de instalação e operação consequentemente também diminuiu com essa crise.

\section{CONCLUSÃO}

Com o crescimento da importância da "Questão Ambiental" nos debates atuais em quaisquer que sejam os fóruns, o setor produtivo se viu obrigado a repensar a sua relação com os recursos do meio ambiente e incorporar o tema em seus processos de tomadas de decisões.

Dessa maneira, o setor de extração petrolífera também tem sentido os novos imperativos que exigem maior responsabilidade ambiental. Tratando-se de uma atividade modificadora do meio ambiente, que causam impactos tanto por explorar um recurso natural, tanto por atingir direta ou indiretamente outros recursos naturais utilizados ou afetados pelo seu desenvolvimento, é necessário que a atividade seja compatibilizada com o meio ambiente.

Sabe-se, contudo, que, se desenvolvida de forma ordenada e sustentável, a atividade petrolífera atrai inúmeros benefícios socioeconômicos, com reduzidos riscos ao meio ambiente, sendo esta uma política já em curso pelos Órgãos de Gestão Ambiental.

O desenvolvimento sustentável deve ser incorporado ao corpo das doutrinas econômicas de desenvolvimento e planejamento, pois este fornece uma multiplicidade de métodos de compreender e investigar em todas suas dimensões. Ao pensar no desenvolvimento sustentável, devem-se aplicar diversas estratégias, tendo sempre como embasamento à flexibilidade, de forma a ser utilizado respeitando as peculiaridades dos diferentes níveis de operação de cada povo.

Portanto, apesar dos problemas causados ao meio ambiente, a atividade é essencial para que a humanidade atinja dois valores sócio-econômicos fundamentais: qualidade de vida e desenvolvimento sustentável. 


\section{REFERÊNCIAS}

BEZERRA, V. L.. LICENCIAMENTO AMBIENTAL DA ATIVIDADE DE REVENDA DE GÁS LIQUEFEITO DE PETRÓLEO (GLP) E SEUS POTENCIAIS IMPACTOS AMBIENTAIS. 2012. $13 \mathrm{f}$. TCC (Graduação) - Curso de Especialização em Perícia e Gestão Ambiental, Centro Universitário do Rio Grande do Norte, Natal, 2012. Disponível em: <http://www2.ifrn.edu.br/ojs/index.php/HOLOS/article/ view/947/569>. Acesso em: 02 dez. 2013.

GIL, Robledo Lima et al. TIPOS DE PESQUISA. 2013. Disponível em: <http://wp.ufpel.edu.br/ecb/ files/2009/09/Tipos-de-Pesquisa.pdf>. Acesso em: 01 out. 2013.

IDEMA. Licenciamento Ambiental: Competência para licenciar. 2013. Disponível em: <http://www.idema. rn.gov.br/Conteudo.asp?TRAN=ITEM\&TARG=2114\&ACT; $=\&$ PAGE $=0 \& P A R M ;=\& L B L=$ Licenciamento+ Ambiental>. Acesso em: 02 dez. 2013.

IDEMA. Licenciamento Ambiental: Tipos de Licenças e Autorizações. 2013. Disponível em: <http://www. idema.rn.gov.br/Conteudo.asp?TRAN=ITEM\&TARG=2115\&ACT; $\&$ PAGE $=0 \&$ PARM $;=\& L B L=$ Licencia mento+Ambiental>. Acesso em: 02 dez. 2013.

LIMEIRA NETO, Manoel Alexandre Diniz. ABORDAGEM DAS FERRAMENTAS DE APOIO AO PROCESSO DE LICENCIAMENTO AMBIENTAL DAS ATIVIDADES DE MINERAÇÃO. 2006. $82 \mathrm{f}$. Monografia (Especialização) - Curso de Especialização em Meio Ambiente e Desenvolvimento Sustentável, Universidade Potiguar, Natal, 2006.

PRODANOV, Cleber Cristiano et al. Ernani Cesar de Freitas: Métodos e Técnicas da Pesquisa e do Trabalho Acadêmico. 2013. Disponível em: <http://tconline.feevale.br/tc/files/06mqxzjogqh/E-book Metodologia do Trabalho Cientifico.pdf>. Acesso em: 05 set. 2013.

PROGEL. Relatório de Controle Ambiental. 2011. 305 f. TCC (Graduação) - Curso de Engenharia de Petróleo e Gás, Petrobras, Natal, 2011.

ROCHA, M. O Cenário Atual do Setor de Petroléo no Rio Grande do Norte ( 1) Refinaria Potiguar Clara Camarão - Rod - RN - 221/S/N - Guamaré/RN. 